\title{
Investigação do uso de plantas medicinais no tratamento de indivíduos com diabetes melito na cidade de Herval D' Oeste - SC
}

ROSA, R.L. ${ }^{*}$; BARCELOS, A.L.V. ${ }^{2}$; BAMPI, G. ${ }^{2}$

${ }^{1}$ Universidade do Oeste de Santa Catarina - UNOESC, Rua Paese, 198, Bairro Universitário, CEP: 89560-000, Videira-Brasil " ${ }^{*}$ roseane.leandra@yahoo.com.br ${ }^{2}$ Universidade do Contestado-UnC, Rua Victor Sopelsa, 3000, Bairro Salete, CEP: 89700-000, Concórdia-Brasil

\begin{abstract}
RESUMO: O diabetes melito é uma doença crônica caracterizada pela elevação da glicose no sangue (hiperglicemia), sendo considerada um distúrbio do metabolismo dos carboidratos, lipídios e proteínas, devido à ausência de insulina produzida pelo pâncreas e/ou pela diminuição de sua ação. Tal patologia é a sexta causa de internação como diagnóstico primário e contribui em até $50 \%$ para outras causas como cardiopatias, acidente vascular cerebral e hipertensão arterial, entre outras. Uma alternativa de tratamento que nos últimos anos vem recebendo aumento de pesquisas e interesse de uso é a fitoterapia, a qual pode ser igualada e somada à terapia convencional. Com isso, o presente estudo teve por objetivo investigar o uso de plantas medicinais no tratamento de indivíduos com diabetes melito na cidade de Herval D'Oeste-SC e também apresentar a importância da ingestão segura de plantas medicinais. Os resultados obtidos mostram que esse consumo de plantas medicinais ocorre de maneira errônea, onde apenas $19 \%$ das plantas citadas tiveram comprovação científica na terapêutica do diabetes. Além disso, grande parte dos usuários de plantas medicinais $(89,4 \%)$ escolhe as espécies vegetais através da cultura familiar. Portanto, há necessidade de mais pesquisas voltadas a essa terapia, assim como, maiores informações para a população e profissionais da saúde em relação à correta utilização das plantas, bem como a forma de preparo e quantidade a ser ingerida.
\end{abstract}

Palavras-chave: diabetes melito, tratamento alternativo/complementar, plantas medicinais, fitoterapia

\begin{abstract}
Research on medicinal plants in treating individuals with diabetes mellitus in the city of Herval D' Oeste, Santa Catarina State, Brazil. Diabetes mellitus is a chronic disease characterized by elevated blood glucose (hyperglycemia) and is considered a disturbance in the metabolism of carbohydrates, lipids and proteins, due to lack of insulin produced by the pancreas and / or reduction in its action. This condition is the sixth leading cause of hospitalization as primary diagnosis and contributes up to $50 \%$ to other causes such as heart diseases, stroke and hypertension, among others. An alternative treatment, which in recent years had an increase in interest and research, is the herbal medicine, which can be equalled and added to conventional therapy. Therefore, this study aimed to investigate the use of medicinal plants in treating individuals with diabetes mellitus in the city of Herval D' Oeste, Santa Catarina State, Brazil and also present the importance of safe intake of medicinal plants. The obtained results showed that consumption of medicinal plants occurs erroneously, where only $19 \%$ of the cited plants had scientific evidence for diabetes therapy. In addition, most users of medicinal plants $(89.4 \%)$ choose the plant species by family culture. Thus, further research focused on this therapy is needed, as well as further information to the public and health professionals regarding the correct use of plants, preparation and quantity to be ingested.
\end{abstract}

Key words: diabetes mellitus, alternative/complementary treatment, medicinal plants, herbal medicine

\section{INTRODUÇÃO}

O diabetes melito (DM) não se apresenta como um distúrbio único no organismo, mas sim, como um grupo heterogêneo que desencadeia a

elevação da glicose sanguínea (hiperglicemia). Isso pode ocorrer devido ao resultado da resistência da ação da insulina à célula, da insuficiência na produção

Recebido para publicação em 04/10/2010

Aceito para publicação em 17/11/2011

Rev. Bras. PI. Med., Botucatu, v.14, n.2, p.306-310, 2012. 
insulínica ou em ambos os casos (Bnouham et al., 2002; Escott-Stump, 2007).

O DM se classifica em dois tipos principais, o diabetes melito tipo I que ocorre pela destruição da célula beta do pâncreas e por decorrência de doença auto-imune o que leva à deficiência de produção de insulina e onde o único tratamento a ser realizado é a aplicação da insulina. O tipo II é causado predominantemente por um estado de resistência a ação da insulina associada à deficiência da secreção (Silva et al., 2008; Moreira \& Chiarello, 2008). Quando não tratados corretamente, ambos os tipos de diabetes poderão desencadear complicações, tais como retinopatia, hipertensão arterial, doenças coronarianas, patologias renais, doenças micro e macro vasculares, entre outras. Assim, mediante essas patologias recorrentes de diabetes melito não tratado e à crescente incidência desta patologia na população com maior abrangência na terceira idade, a demanda por terapias que tenham menor custo podem representar um benefício significativo para os investimentos empregados na área de saúde (Cetto \& Heinrich, 2005; Moreira \& Chiarello, 2008).

Nos últimos anos, uma alternativa de tratamento despertou o interesse de pesquisadores e com isso aumentaram-se as pesquisas referentes às plantas medicinais. O termo "fitoterapia" resulta da junção das palavras gregas "Phythón" - planta - e "Therapeía" - terapia - ou seja, terapia com as plantas, a qual visa o estudo de plantas e as aplicações no tratamento de patologias (Teske \& Trentini, 2001; Kalluf, 2008).

A fitoterapia é uma área ampla, sendo necessário para a melhor compreensão conhecer algumas definições importantes que a envolvem. Primeiramente, é importante diferenciar planta medicinal de fitoterápico. Para Veiga Junior e colaboradores (2005) a planta medicinal é "todo e qualquer vegetal que possui, em um ou mais órgãos, substâncias que podem ser utilizadas com fins terapêuticos ou que sejam precursores de fármacos semi-sintéticos". O fitoterápico, por sua vez, é um medicamento tecnicamente obtido, com formulação específica, empregando-se apenas matéria prima de fonte vegetal, cujo efeito tenha sido comprovado cientificamente (Kalluf, 2008).

Também, é necessário, neste ínterim, destacar a droga vegetal, que é a planta inteira, ou as partes, que após a coleta, secagem, estabilização e conservação, passam a ser utilizadas na preparação de medicamentos, considerando que o princípio ativo é o responsável pelos efeitos terapêuticos, tanto das plantas in natura, na forma de droga vegetal ou nas diferentes formas farmacêuticas (Kalluf, 2008; Brasil, 2010).

Além das definições citadas, as plantas medicinais envolvem modos diferentes de preparo, merecendo destaque as formas que podem ser prescritas pelos nutricionistas, como a infusão, a decocção, a tintura, a alcoolatura e o extrato. (Conselho Federal de Nutricionistas, 2007).

Ainda, dentro deste contexto, a Agência Nacional de Vigilância Sanitária (2005) caracterizou o chá como "o produto constituído de uma ou mais partes de espécie(s) vegetal(is) inteira(s), fragmentada(s) ou moída(s), com ou sem fermentação, tostada(s) ou não", considerando que, de maneira geral, todos os órgãos das plantas podem servir como ingredientes para chás, sempre distinguindo as plantas meramente aromáticas, das medicinais e de ambas. $O$ chá pode ser considerado uma rica fonte de flavonóides e pode ser utilizado procurando-se obter benefícios das propriedades, desde que se conheça a quantidade e a maneira de utilizá-la, já que cada planta tem uma maneira correta de utilização (Rhomer, 2002; Kalluf, 2008).

As plantas medicinais representam atualmente a origem de $25 \%$ dos fármacos utilizados, sendo que isto se deve a grande flora mundial e as importantes propriedades terapêuticas conhecidas. No Brasil, várias são as pesquisas realizadas que vem contribuindo significativamente para o desenvolvimento e uso destas espécies vegetais, pois vem comprovando efeitos terapêuticos, possibilitando a utilização destas para tratamento de patologias (Shu, 2005; Kalluf, 2008).

Conforme estudos científicos realizados com plantas medicinais ao longo dos anos, algumas plantas medicinais já receberam confirmação das ações terapêuticas hipoglicemiantes, sendo as mais citadas: Baccharis trimera (Less.) DC. (carqueja), Bauhinia forficata Link. (pata de vaca), Salvia Officinalis L. (salvia), Mormodica charantia L. (melão de São Caetano), Phyllantus niruri L. (quebra-pedra) e Myrcia sphaerocarpa DC. (insulina vegetal) (Grover et al., 2002; Lino et al., 2004; Barbosa et al., 2005; Sathishsekar \& Sorimuthus, 2005; Borges et al., 2008; Cunha et al., 2010).

Nesse contexto, o presente estudo investigou o uso de plantas medicinais utilizadas no tratamento de indivíduos com diabetes na cidade de Herval d' Oeste - SC, verificando: o modo de preparo e o tempo de consumo das espécies vegetais, a utilização dessas de forma concomitante ao uso de medicamentos, e a fonte das orientações buscando demonstrar que a utilização das plantas medicinais ocorre de maneia incorreta, passível de ocasionar efeitos indesejáveis aos usuários.

\section{MATERIAL E MÉTODO}

Foram avaliados 73 indivíduos moradores da cidade de Herval D' Oeste - SC ( $n=73)$, tendo como critérios de inclusão serem diabéticos, maiores de 
18 anos e de ambos os sexos. A coleta de dados realizou-se a domicílio através de questionário estruturado, o qual continha pergunta sobre a utilização de plantas medicinais, o uso de medicamentos concomitantes às plantas, a fonte de orientação do consumo e origem destas plantas. Os resultados do estudo foram observados em gráficos do Microsoft Excel e comparados com as referências pesquisadas. Cabe ressaltar que o pesquisador somente respondeu os questionários de indivíduos analfabetos. Esta pesquisa foi aprovada pelo Comitê de Ética em Pesquisa da Universidade do Oeste de Santa Catarina - UNOESC sob o Parecer no 173/09.

\section{RESULTADO E DISCUSSÃO}

Entre os participantes da pesquisa $22,7 \%$ (10) possuíam o diabetes melito tipo I, os outros $86,4 \%$ (63) possuíam o tipo II; $29,7 \%$ (21) eram do sexo masculino e 70,3\% (52) eram do sexo feminino. Nessa população, $55,4 \%$ (40) tinham mais de 60 anos e 44,6\% (33) menos de 60 anos. Pode-se observar também que a maior prevalência foi do diabetes tipo II, sendo mais frequente na população feminina e idosa, características essas que coincidem com os achados literários, pois, segundo Teixeira (2003), o diabetes tipo II acomete aproximadamente $90 \%$ dos casos de diabetes melito e na maioria, mulheres e idosas.

No que tange ao uso da terapia com plantas medicinais, dos 73 indivíduos pesquisados, $59,4 \%$ (43) consumiam plantas medicinais como forma de tratamento do diabetes, dados esses que se comparam ao estudo de Pinto (2008), onde $60 \%$ da população pesquisada utilizavam plantas medicinais para tratamento de patologias.

Da população que relatou ingerir plantas medicinais, $81,8 \%$ (36) eram do sexo feminino e $18,2 \%$ (7) do sexo masculino, sendo que $63,4 \%$ (26) desses tinham mais de 60 anos e 53,1\% (17) menos de 60 anos. Esses dados apóiam os resultados da pesquisa de Borges e colaboradores (2008), que demonstram o sexo feminino como o mais propenso a desenvolver o diabetes tipo II e que ainda é o maior consumidor de plantas medicinais.

A utilização de plantas medicinais foi realizada em sua maioria por indivíduos idosos. Sugere-se que este fato ocorra, possivelmente, devido à cultura familiar de optar pelo tratamento com plantas como a primeira escolha. Pilla e colaboradores (2006) em pesquisa realizada em São Paulo, verificaram o consumo de plantas medicinais em $60 \%$ dos indivíduos idosos.

A utilização de plantas medicinais é embasada em grande parte na cultura dos povos, como obtido também no presente estudo, onde $89,3 \%$ (38) dos indivíduos passam a utilizar as plantas medicinais como terapia mediante indicação de familiares (citam tias, vizinhas, amigas). Apenas 4,6\% as consomem com instrução científica advinda de livros e/ou por indicação médica. A mídia (principalmente a televisão) foi um item relatado por $6,9 \%$ dos indivíduos.

Também pode-se verificar que o uso de plantas medicinais é concomitante ao de medicamentos em 93,1\% (40) dos participantes. Diante disso, ressalta-se que a interação entre drogas e plantas medicinais pode diminuir ou potencializar os efeitos de ambos (Alexandre et al., 2008).

Em relação ao período de consumo de plantas medicinais, em $72 \%$ (31) dos casos, os indivíduos ingeriam as plantas há mais de um mêse em $25,5 \%$ (11) há um mês, aproximadamente. Quanto à frequência de consumo, $58,1 \%(25)$ consumiam de vez em quando, 20,9\% (9) consumiam de cinco a sete vezes na semana e $20,9 \%$ (9) consumiam diariamente, como forma de tratamento. Isso demonstrou desorientação da população no consumo de plantas medicinais, pois a dosagem terapêutica, a frequência de consumo, e o tempo de administração para obter-se resultado no tratamento são particulares de cada planta medicinal e, consequentemente, de seus componentes bioativos que assim como os medicamentos alopáticos necessitam de período de uso para realizarem os efeitos terapêuticos (Kalluf, 2008).

Quanto a origem das plantas, $60,4 \%$ (26) são adquiridas através de plantio próprio, já 30,3\% (13) são nativas e 9,3\% (4) são adquiridas no comércio local (farmácias de homeopatia, supermercados e lojas de produtos naturais).

Quando cultivadas em casa, o plantio das plantas deve ser monitorado, já que as plantas podem desenvolver fungos que causam intoxicação, assim como as nativas que podem conter agrotóxicos prejudiciais à saúde (Alexandre et al., 2008). Os dados da presente pesquisa coincidem com o estudo de Pilla e colaboradores (2006), que relatam ser o quintal das casas o local mais citado pelos indivíduos como fonte de obtenção das espécies vegetais.

Em $88,3 \%$ dos casos, a parte mais utilizada das plantas para as preparações foi a folha. Dados que corroboram com a pesquisa de Borges e colaboradores (2008), que apresentaram a preferência pela folha em $38 \%$ dos casos levantados, e que representavam a maioria. A prática mais comum de preparação relatada foi a infusão, em quase $70 \%$ dos casos, e a decocção, nos $30 \%$ restantes. Esse resultado também foi observado por Arnous e colaboradores (2005) que verificaram que $75,2 \%$ dos indivíduos entrevistados confirmaram a preferência pela infusão como forma de preparo das plantas medicinais.

Foram citadas 21 espécies de plantas 
medicinais, dessas, $81 \%$ apresentam relato na literatura de que não têm efeito terapêutico para o diabetes. De acordo com as pesquisas científicas, apenas $19 \%$ das plantas citadas têm respaldo da comprovação científica sobre o efeito terapêutico hipoglicemiante potencial para o tratamento do diabetes, são elas: a Bauhinia forficata Link. (pata de vaca); Baccharis trimera (Less.) DC. (carqueja); Myrcia sphaerocarpa DC. (insulina vegetal); Phyllantus niruri L. (quebra pedra).

É preocupante observar que somente $48,8 \%$ dos entrevistados utiliza plantas com comprovação científica de efeito hipoglicemiante, enquanto $51,2 \%$ dos indivíduos consomem plantas que não tem ação terapêutica comprovada para o diabetes. Esse dado possibilita constatar que é alto o consumo de plantas medicinais pela população com diabetes da cidade de Herval d' Oeste; no entanto, este consumo é feito, em grande parte, de maneira errônea sem a devida orientação sobre a utilização.

Observa-se a necessidade da inclusão do conhecimento de terapias alternativas, como a fitoterapia, no cotidiano de profissionais da saúde. Essa inclusão abre maiores possibilidades para que estes profissionais realizem as atividades em caráter multidisciplinar, buscando melhorar a saúde da população e a qualidade de vida da mesma, proporcionando novas opções de tratamento para as patologias.

Assim, diante do crescimento constante desta terapêutica, da adesão da população, e visando agregar novos campos para atuação do nutricionista na busca de melhorias para o planejamento da promoção da saúde do ser humano, o CFN formulou a Resolução CFN número 402 de 2007 que "regulamenta a prescrição fitoterápica pelo nutricionista de plantas in natura frescas, ou como droga vegetal nas suas diferentes formas farmacêuticas, e dá outras providências" (Conselho Federal de Nutricionistas, 2007).

Os nutricionistas vêm apostando e atuando nesta área através da prescrição, dentro das normalizações da ANVISA e do CFN, da plantas medicinais in natura fresca ou como droga vegetal nas diferentes formas farmacêuticas, que possam auxiliar em casos de obesidade, síndrome metabólica e/ou doenças associadas decorrentes de desordens na alimentação. Também vêm atuando na disseminação do conhecimento para a população que busca melhorar a qualidade de vida, evitando o consumo exacerbado e errôneo dessas espécies vegetais, podendo assim evitar intoxicações e efeitos adversos.

Uma vez que o consumo de plantas medicinais in natura fresca ou como droga vegetal nas diferentes formas farmacêuticas pode auxiliar a população na prevenção e tratamento de patologias, percebe-se a necessidade de maior conhecimento dos profissionais da saúde, para que estes possam orientar e/ou recomendar um profissional habilitado para as indicações e prescrições corretas à população.

\section{REFERÊNCIA}

AFOLAYAN, A.; SUNMONUT, T.O. In vivo studies on antidiabetic plants used in South African herbal medicine, Journal Clinical Biochemistryand Nutrition, v.47, p.98106, 2010.

ALBUQUERQUE, R. Últimas novidades do Congresso Americano de diabetes. Sociedade Brasileira de Diabetes. 2009. Disponível em: <http://www.diabetes. org.br>. Acesso em: 15 out. 2009

ALBUQUERQUE, U.P.; OLIVEIRA, R.F. Is the use-impact on native caatinga species in Brazil reduced by the high species richness of medicinal plants? Journal of Ethnopharmacology, v.113, n.1, p.156-70, 2007.

ALEXANDRE, R.F. et al. Interação entre fármacos e medicamentos fitoterápicos a base de ginko ou ginseng. Revista Brasileira de Farmacognosia, v.1, n.18, p.11726, 2008.

AMERICAN DIABETES ASSOCIATION. Nutritional recommendation in diabetes. Diabetes Care, v.1, n.27, p.36-4, 2004. Disponível em: <http://care. diabetesjournals.org>. Acesso em: 09 nov. 2009.

ARNOUS, A.H. et al. Plantas medicinais de uso caseiro: conhecimento popular e incentivo por cultivo comunitário.

Revista Espaço para a Saúde, v.6, n.2, p.1-6, 2005.

BALDAUF, C. et al. "Ferveu, queimou o ser da erva": conhecimentos de especialistas locais sobre plantas medicinais na região Sul do Brasil. Revista Brasileira de Plantas Medicinais, v.11, n.3, p.282-91, 2009.

BARBOSA, J.B.F. et al. Plants and their active constituintes from South, Central and North America with hypoglycemic activity. Revista Brasileira de Farmacognosia, v.15, n.4, p.392-413, 2005.

BNOUHAM, M. et al. Medicinal plants used in the treatment of diabetes in Morocco. Journal International Diabete \& Metabolism, v.10, p.33-50, 2002.

BORGES, K.B. et al. Diabetes - utilização de plantas medicinais como forma opcional de tratamento. Revista Eletrônica de Farmácia, v.5, p.12-20, 2008.

BRASIL, Resolução no 17, de 24 de fevereiro de 2000. Dispõe sobre o registro de medicamentos fitoterápicos. Agência Nacional de Vigilância Sanitária. Brasília: Ministério da Saúde, 2000. 146p.

BRASIL, Resolução no 277, de 22 de setembro de 2005. Dispõe sobre o regulamento técnico para café, cevada, chá, erva-mate e produtos solúveis. Agência Nacional de Vigilância Sanitária. Brasília: Ministério da Saúde, 2005. Disponível em: <http://www.anvisa.gov.br>. Acesso em: 01 jul. 2011.

BRASIL, Resolução no 10, de 9 de março de 2010. Dispõe sobre a notificação de drogas vegetais junto à Agência Nacional de Vigilância Sanitária (ANVISA) e dá outras providências. Agência Nacional de Vigilância Sanitária. Brasília: Ministério da Saúde, 2010. Disponível em: <http:// bvsms.saude.gov.br/bvs/saudelegis/anvisa/2010/>. Acesso em: 20 jan. 2011. 
CARVALHO, J.C.T. Fitoterápicos anti-inflamtórios: aspectos químicos, farmacológicos e aplicações terapêuticas. Ribeirão Preto: Tecmedd, 2004. 286p.

CETTO, A.A.; HEINRICH, M. Mexican plants with hypoglycemic effect used in the treatment of diabetes. Journal of Ethnopharmacology, v.99, p.325-48, 2005. CONSELHO FEDERAL DE NUTRICIONISTAS. Regulamenta a prescrição fitoterápica pelo nutricionista de plantas in natura frescas, ou como droga vegetal nas suas diferentes formas farmacêuticas, e dá outras providências. Resolução n. 402 de 30 de julho de 2007. Lex: Conselho Federal de Nutricionistas. Disponível em: <http://www.cfn.org.br/novosite/pdf/res/2007/res402. pdf>. Acesso em: 23 fev. 2009.

CORREA, A.D.; BATISTA, R.S.; QUINTAS L.E.M. Plantas medicinais: do cultivo a terapêutica. 4.ed. Rio de Janeiro: Vozes, 2001. 284p.

CUNHA, A.M. et al. Hypoglycemic activity of dried extracts of Bauhinia forficata Link. Journal Phytomedicine, v.17, n.1, p.37-41, 2010.

CUNHA, S.T.L. et al. Levantamento da flora medicinal usada no tratamento de doenças metabólicas em Salvador BA - Brasil. Revista Brasileira de Plantas Medicinais, v.10, n.4, p.83-9, 2008.

ESCOTT-STUMP, S. Nutrição relacionada ao diagnóstico e tratamento. 5.ed. São Paulo: Manole, 2007. 847p.

ERASTO, P. An ethnobotanical study of plants used for the treatment of diabetes in the Easten Cape Province, South Africa. African Journal of Biotechnology, v.4, n.12, p.1458-60, 2005.

GROVER, J.K. et al. Medicinal plants of India with antidiabetic potential. Journal of Ethnopharmacology, v.80, p.81-100, 2002.

KALLUF, L.J.H. Fitoterapia funcional: dos princípios ativos à prescrição de fitoterápicos. 1.ed. São Paulo: VP Editora, 2008. 304p.

LIMA, G.R. Proposta de resolução específica para manipulação de plantas medicinais e fitoterápicos. 2006. 40p. Monografia (Pós Graduação em Ciências da Saúde) - Universidade de Brasília, Goiás.

LINO, C.S. et al. Antidiabetic activity of Bauhinia forficata extracts in alloxon diabetic rats. Biological \& Pharmaceutical Bulletin, v.27, n.1125, p.125-27, 2004. Disponível em: <http://www.ncbi.nlm.nih.gov/pubmed/>. Acesso em: 02 abr. 2011.

LORENZI, H.; MATOS, F.J.A. Plantas medicinais no Brasil: nativas e exóticas cultivadas. Nova Odessa, Instituto Plantarum, 2002. 382p.

MACEDO, A.F. et al. Ocorrência do uso de plantas medicinais por moradores de um bairro do município de Marília-SP. Revista de Ciências Farmacêuticas Básica e Aplicada, v.28, n.1, p.123-8, 2007.

MANTENA, S.K. et al. Antiallergic, antipyretic, hypoglycemic and hepatoprotective effects of aqueous extract of Coronopus didymus LINN. Biological \& Pharmaceutical Bulletin, v.28, n.3, p.468-73, 2005. Disponível em: <http://sciencelinks.jp/j-east/>. Acesso em: 03 abr. 2011.

MODAK, M. et al. Indian herbs and herbal drugs used for the treatment of diabetes. Journal Clinical Biochemistryand Nutrition, v.40, n.3, p.1-11, 2007.

MOREIRA, A.M.; CHIARELLO, P.G. Atenção nutricional: abordagem dietoterápica em adultos. Rio de Janeiro: Guanabara Koogan, 2008. 220p.

NOLLA, D. et al. Plantas medicinais. 2.ed. Passo Fundo: UPF, 2005. 72p.

JUNG, M. et al. Antidiabetic agents from medicinal plants. Bentham Science Publishers, v.13, n.10, p.1203-18, 2006. PILLA, M.A.C. et al. Obtenção e uso das plantas medicinais no Distrito de Martin Francisco, Município de Mogi Morim, SP, Brasil. Acta Botanica Brasilica, v.20, n.4, p.789-802, 2006.

PINTO, L.N. Plantas medicinais utilizadas no município de Igarapé Miri, Pará. 2008. 112p. Tese (Pós Graduação em Ciências Farmacêuticas) - Universidade Federal do Pará, Belém.

RHOMER, F. O Livro do chá. São Paulo: Aquariana, 2002. $144 p$.

SATHISHSEKAR, D.; SORIMUTHUS, S. Beneficial efects of Momordica charantia seeds in the treatment al STZinduced diabetes in experimental rats. Biological Pharmaceutical Bulletin, v.28, n.6, p.978-83, 2005. Disponível em: <http://ukpmc.ac.uk/abstract/MED/>. Acesso em: 05 jan. 2010

SILVA, C.L. et al. A expansão do diabetes e o uso de fitoterápicos extraído das plantas do gênero Bauhinia. Revista Digital de Pesquisa CONQUER da Faculdade São Francisco de Barreiras, v.3, 2008. Disponível em: <http://www.fasb.edu.br/revista/index.php/conquer/ article/view/98/76>. Acesso em: 15 fev. 2009.

SHU, Y.Z. Recent natural products based drug development: a pharmaceutical industry perspective. Journal of Natural Products, v.61, p.1053-71, 2005. TEIXEIRA, F.N. Nutrição clínica. Rio de Janeiro: Guanabara Koogan, 2003. 550p.

TESKE, M.; TRENTINI, A.M. Herbarium compêndio de fitoterapia. 4.ed. Curitiba: Herbarium Laboratório Botânico Ltda, 2001. 318p.

VEIGA JUNIOR, V.F. et al. Plantas medicinais: cura segura? Revista Química Nova, v.28, n.3, p.519-28, 2005.

Rev. Bras. PI. Med., Botucatu, v.14, n.2, p.306-310, 2012. 